\title{
Seeing goal-directedness: a case for social perception
}

\author{
Joulia Smortchkova
}

\begin{abstract}
This paper focuses on social perception, an area of research that lies at the interface between the philosophy of perception and the scientific investigation of human social cognition. Some philosophers and psychologists appeal to resonance mechanisms to show that intentional and goal-directed actions can be perceived. Against these approaches, I show that there is a class of simple goal-directed actions, whose perception does not rely on resonance. I discuss the role of the STS (superior temporal sulcus) as the possible neural correlate of perception of goal-directed actions. My proposal is intermediate between claims according to which we perceive intentional actions and claims according to which we cannot perceive goal-directed actions.
\end{abstract}

1. Preliminary clarifications and methodology ......................................................... 1

2. Does the perception of goal-directed actions rest on resonance? ................................ 7

3. What are the neural correlates of social perception (without resonance)? ................. 15

4. From neural correlates to the experience of goal-directed actions ............................. 22

5. The development of the perception of goal-directed actions: A possible new role for

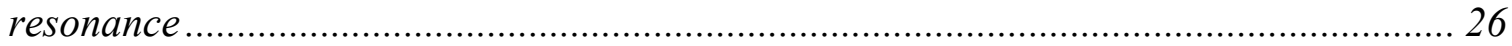

6. Conclusion: seeing goal-directedness of actions and social perception ..................... 31

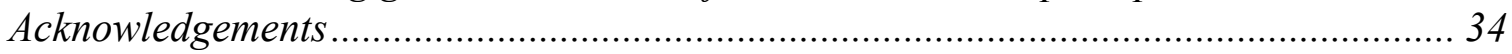

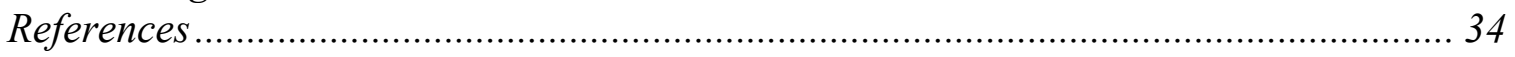

\section{Preliminary Clarifications AND METhOdOLOGY}

The topic of this paper lies at the interface between the philosophy of perception and the 
scientific investigation of human social cognition, the study of how we apprehend (that is, perceive or understand) other people. In recent cognitive science, the term 'social perception' has come to refer to the initial visual stages of processing of information relevant to human social cognition (Allison, Puce and McCarthy [2000]). Much experimental work in social perception focuses on the existence of brain mechanisms dedicated to the perception of human bodies and human faces, and to the initial stages of processing of socially relevant information.

In this paper, I focus on one potential case of social perception and its relation to social understanding: perception of goal-directed actions. This topic is also relevant to broader concerns in philosophy of perception about the reach of perceptual content (Hawley and Macpherson [2011]). While it is widely agreed that we can see the shape, size, orientation, color, ... of objects, it is a matter of debate whether we can also see, and not merely cognize or conceptually represent, higher-level and complex properties, such as causation, being a pine tree, or being a forest (Hawley and Macpherson [2011]). In the context of social perception, while it is uncontroversial that we can see others' bodily parts and movements, I want to argue that the high-level, complex property of being a goal-directed action can be represented in perception, and not merely cognized or conceptually represented. In other words, we can see goal-directedness.

The paper is structured as follows. In section 1, I introduce some central notions, outline the issues at the core of the paper, and clarify its methodology. In section 2, I consider and criticize the view according to which the perception of goal-directed actions rests on resonance. These criticisms help to make room for the introduction of my positive view, which I present in section 3. There I provide some empirical evidence which shows that 
there is social perception of goal-directed actions in the absence of resonance. This evidence comes from different sources: studies devoted to the neural circuits underlying the perception of goal-directed actions and psychophysical studies in adults. In section 4 I tentatively connect the discussion of the neural correlates of goal-directed action perception with perceptual experience of goal-directed actions. In section 5, I further flesh out this thesis, by exploring a hypothesis about the developmental trajectory of our capacity to visually perceive actions.

Before launching into the main argument of this paper, I need to make two terminological clarifications. First, I distinguish different meanings of 'goal'. Second, I clarify my use of the term 'goal-directed action', which is based on the way this notion is employed in the empirical literature.

When talking about goals, we can use the term in at least two ways. 'Goal' can be used to refer to complex mental states involving long term plans and aimed at fulfilling one's aims, embedded in a net of related psychological states (for example when we say: 'Her goal is to become the next President.'). On this reading, goals cannot be perceived because they are private psychological states of the agent and are deeply interwoven in a complex web of intentions, beliefs, and desires in the mind of the agent who has the goal.

In the case of visible goal-directed actions, 'goal' can be used in a simpler, deflationary way, to indicate the outcome of a visible movement, whether or not the movement is guided by a mental state. In this external sense, goals are individuated perceptually as the end-points of visible trajectories, and not via the presence of an underlying teleological mental state. In the psychological literature, the outcomes (goals) of actions are events, 
which can involve objects (grasping an apple, touching a doll, ...), locations and objects (placing the cup in a certain location, ...) or just locations (sprinting to some place, ...). Henceforth, unless otherwise specified, I use 'goal' in this deflationary sense to mean the outcome of a trajectory.

Secondly, I use the term 'goal-directed actions' as it is usually employed in the empirical literature on social cognition. By this term I mean a movement directed toward a goal, and not an intentional action as that notion is often used in the philosophy of action. ${ }^{1}$ In the sense used in this paper, a goal-directed action is an efficient motoric means whereby an agent achieves its outcome given the situational context, whether or not the agent really intended to execute the action (let alone has full-blooded mental states). In this cognitively less demanding sense, the agent is any entity that exhibits perceptual cues associated with agency: self-propelled motion, contingent movements in response to the environment, ... (Premack [1990]). I focus on a limited class of simple goal-directed actions that can be analyzed into their perceptual components, as comprising the visually presented target (goal), the spatiotemporal path to the goal, the entity moving to the goal, and the visually presented obstacles (if there are any).

Nevertheless, it is controversial that even the minimal notion of goal-directed action outlined above can be perceived. Indeed, it has been suggested that goal-directed actions can be apprehended only in cognition: 'The motions [of a hand towards an object] could

${ }^{1}$ In the philosophy of action, goal-directed actions are a case of intentional actions, defined with respect to the presence or absence of an underlying propositional attitude, an intention (Davidson [1963]; Searle [1983]). The presence of an underlying intention is what distinguishes, say, breathing, from switching the light on. Philosophers connect intentions to reasons, so that knowing that a person has done something intentionally requires knowing that the person had some reasons to act this way. 
be characterized entirely in spatiotemporal terms - in terms of the trajectories of each figure relative to the others, and to those of each other. Nowhere in these descriptions would we find concepts such as goal.' (Carey [2009], p. 171). The idea behind this quote is that in order to represent a movement as goal-directed one needs to possess and apply the concept GOAL.

I will defend the contrasting view according to which there is perceptual processing of goal-directedness. The perception of goal-directedness would clearly a case of 'rich' perception, that is, perception of higher-level properties. Thus, the overarching question that motivates this paper is: What is the relation between the reach of perceptual content and human social cognition? In particular, can some of the properties that we are intuitively inclined to call 'social' be represented in perception?

Bringing together the debate about the reach of perception and the debate about the nature of basic social cognition, I will argue that being a goal-directed action performed by another can indeed be represented in perception. In other words, I will argue that the property of an action of being an efficient means for achieving the agent's goal can be encoded in the content of vision. Since most goal-directed actions which we perceive are performed by other human beings, and perceiving these actions plays a major role in shaping our social interactions, this is highly relevant to human social cognition more generally.

A point of methodology: In order to extend the reach of perceptual content, it is customary to start from perceptual experiences and 'seeming states,' which are taken to non-problematically encode low-level sensory properties, and try to extend the reach of 
the content of perception to higher-level properties, whose representation may seem prima facie to require the possession of conceptual resources, while appealing exclusively to introspectable phenomenological intuitions (Siegel [2010]). This strategy rests on the assumption that there is an introspectable difference between seeing something while experiencing it as an $\mathrm{F}$ and seeing something without experiencing it as an $\mathrm{F}$, where $\mathrm{F}$ is taken to be a high-level property (such as being a pine tree). The goal of the strategy is to account for the purported introspectable experiential difference by an inference to the best explanation. The best explanation in this case would purportedly be that the phenomenal contrast is due to the presence of a visual non-cognitive phenomenology.

Because it rests on several assumptions, which I take to be contentious, I prefer to appeal to a different strategy: basing my claim on evidence about the underlying psychological and neural mechanisms, I argue that perception can represent goal-directed actions. I do not focus solely on the phenomenology of perceptual experiences ${ }^{2}$ to argue that goaldirected actions can be perceptually represented. Instead, I appeal to empirical evidence from neuroscience and vision science in support of the phenomenological resources.

There are two reasons for this choice. First, it might be that conscious content and perceptual content do not perfectly align (Burge [2010], pp. 34-46). Perceptual content can play a role in a person's cognitive life despite not being consciously accessed, for example by guiding on-line reactions in the absence of recognition. This might be particularly true of social perception, where we can react to some social features of the world even before recognizing that they are. The second reason is a methodological one: 
while the point about whether goal-directed actions can be represented in perception is mainly conceptual, it obviously bears on the interpretation of experimental data from social cognitive neuroscience, and psychophysical studies in adults. Therefore, I appeal to studies in vision science that are not always based on conscious experiences.

\section{DOES THE PERCEPTION OF GOAL-DIRECTED ACTIONS REST ON RESO- NANCE?}

Some scientists and philosophers have argued that perception cannot represent goaldirected actions on the grounds that perception can only represent low-level sensory properties (for example Carey [2009], p. 171). In the debate on direct social perception, Spaulding has argued that the claim that we can perceive others' mental states (including goal-directed actions) just like we perceive ordinary objects and without the application of cognitive inferences is implausible (Spaulding [2015]). Drawing on Dretske's distinction between simply seeing and epistemic seeing, she suggests that we can epistemically see others' mental states. But to epistemically see that someone is happy, one has to possess concepts for mental states (such as the concept HAPPINESS) (Spaulding [2015]). Epistemic seeing is not a case of perceptual experience, but a case of belief, formed on the basis of perception: it is thus a cognitive, and not a perceptual, state.

Recently, a competing view has gained traction among philosophers and cognitive scientists: they suggest that we can perceptually represent not merely goal-directed actions, but others' intentions (that is others' mental states) as well (Pacherie [2005]; 
Proust [2003]; Gallese [2009]). ${ }^{3}$

The claim is that we perceive intentional actions via a mechanism called 'resonance' (Knoblich [2008]; Pacherie [2005]; Proust [2003]; Gallese [2009]). Resonance is broadly defined as the mapping of another's action onto one's own motor repertoire. According to the resonance research program, perceptual resonant processes underlie our appraisal of others' observed actions (Pacherie [2005]; Rizzolatti and Sinigaglia [2007]): resonant mechanisms are activated both when we execute an action, and when we observe an action performed by someone else. Resonant theories postulate that perception and action share a common computational code (Prinz [1990]), which means that actions are coded in terms of the perceivable effects they generate. These codes are available both during action performance in oneself and during action observation in others.

One potential source of empirical support for such views is the discovery of mirror neurons (neurons active both during execution of an action and observation of an action performed by another), which has been widely taken to show that the very same resources necessary for executing goal-directed actions are sufficient for understanding and even perceiving the same kinds of actions performed by others (Rizzolatti and Sinigaglia [2007]). Thus, the discovery of mirror neurons ${ }^{4}$ in the primate brain has been

\footnotetext{
${ }^{3}$ Direct social perception approaches often claim that perception is an alternative (or additional) route to mindreading for understanding others (Kiverstein [2015]; Krueger [2012]). Defenders of social perception appeal both to phenomenological considerations and to empirical studies on perception to show that we can directly perceive some mental states without the need for a cognitive, high-level process. With such defenders of social perception, I share two ideas: that we can perceive some social properties, and that we can show this via an appeal to empirical data. Nevertheless, I disagree with most direct social perception approaches with respect to commitment to the enactivist and embodiment theses (on which I am neutral).

4 Mirroring refers to a ' $[\mathrm{n}]$ eural process $\mathrm{N}$ [that] is an activation of a mirror neuron or mirror system, and (2) $[\ldots]$ results from observing something that is normally a behavioral or expressive manifestation (or a predictive sign) of a matching mirror event in another individual.' (Goldman [2013], p. 91). A 'mirror system' is a 'neural system [...] that gets activated both when an individual undergoes a certain mental or
} 
interpreted as the discovery of a mechanism whereby the observer's brain resonates with the agent's brain: the perception of the agent's overt action automatically causes the observer to covertly map the agent's executed movements onto the observer's motor repertoire without executing it (Rizzolatti et al. [2001]).

For instance, Rizzolatti and Sinigaglia write: 'We immediately recognize a specific action. [The monkey] immediately perceives the meaning of these motor events and interprets them in terms of an intentional act' (Rizzolatti and Sinigaglia [2008], p. 9798).

Gallese expresses a similar idea: 'To see others' behavior as an 'action' [...] specifically requires such behaviors to be mapped according to an isomorphic format. Such mapping is embodied simulation' (Gallese [2009], p. 527).

Pacherie writes: 'The purpose of this paper is to examine under what conditions it makes sense to say that we can perceive actions performed by other agents as intentional and, thus, in a sense, can perceive intentions' (Pacherie [2005], p. 2).

I will follow resonance theorists in arguing that our access to others' actions is perceptual in nature, but in contrast to resonance theory, I will argue that, while resonance plays some role in influencing how we perceive actions, it is not necessary to perceive goaldirectedness in the first place. Two distinct questions are raised by the claims of the resonance theory, both of which are controversial and rarely distinguished.

The first question (the representational scope question) is whether resonance enables an

cognitive event endogenously and when he observes a sign that another individual is undergoing, or is about to undergo, the same type of mental or cognitive event.' (Goldman [2013], p.91). 
observer to represent (and understand), not merely an agent's goal-directed action (whereby, for example, the agent reaches for and grasps a target), but the content of her intention (that is a psychological state) as well.

The second question (the mechanisms question) is whether resonance mechanisms should be construed as necessary and sufficient conditions for perceiving others' goal-directed actions, that is whether resonance mechanisms provide grounds for the view that one can perceive, not merely others' bodily movements, but their goal-directed actions as well.

I will quickly address the first question, which has already been extensively debated (see Spaulding [2013]; Jacob [2009]). ${ }^{5}$ The crux of the debate is whether the representational resources postulated by resonance theorists are sufficient to represent another's intention, beyond what is visible: according to one interpretation proposed by Spaulding, resonance causally (and not constitutively) contributes to understanding of goal-directed behaviors only (Spaulding [2013]).

An agent's goal-directed action (or behavior) includes several components, ranging from the agent's body, her bodily movements, and their effects on the immediate environment surrounding the agent's body, in particular on the target of her bodily movements, all of which can be perceptually tracked. Assuming the existence of resonance mechanisms in the observer's brain (that is of mirror neurons or the mirror system for actions), they likely enable the observer to map (or match) the agent's bodily movements onto her own

5 The different interpretations of resonance mechanisms are: resonance is constitutive of mindreading (Gallese [2009]), resonance causally contributes to low-level mindreading (Goldman [2013]), resonance allows for the perception of intentions (Pacherie [2005]), resonance causally contributes to behavior reading (Spaulding [2013]), resonance is involved in processing that follows mindreading (Jacob [2009]), resonance is enactive social perception (Gallagher [2008]). The correct interpretation of mirroring and resonance in respect to mindreading does not concern me in this paper. 
motor repertoire, without executing them.

The question now arises: by mapping the agent's movements onto her motor repertoire, could resonance enable the observer to represent the agent's goal? Could it enable the observer to represent her bodily movements as an efficient means towards achieving her goal? Or else, could it enable the observer to represent the agent's intentions? If so, then what type of intentions would they represent? Arguably, an agent's intentions form a hierarchy ranging from executive intentions to prior intentions. ${ }^{6}$ But all of an agent's intentions, including executive intentions, are private mental states. To access another's intention in order to use it for prediction and explanation of another's behavior, one plausibly needs to go beyond overt behaviors to somehow access others' mental states. The question that now arises is how to bridge the gap between seeing an agent's overt movements and seeing her private intentions. Resonance mechanisms are put to use in order to bridge this gap. Yet, there are several obstacles facing the endorsement of resonance as a mechanism for mindreading and intention understanding (see also Catmur [2015]). So, the first issue concerns the contribution of resonance mechanisms to mindreading (that is to conceptually understanding) an agent's intentions, and I will now set it aside to focus on the question of perception.

The second issue, to which I now turn, concerns the perceivability of goal-directedness in visible actions. A second claim often made on behalf of resonance mechanisms (and which is most relevant to this paper) is that they are necessary conditions for the

\footnotetext{
${ }^{6}$ Intentions can form hierarchies of complexity, ranging from future directed intentions (my intention to bake a cake), to present intentions (my intention to make the batter), to intentions in action or executive intentions (the intention underlying the bodily act of whisking egg whites) (Searle [1983]).
} 
perception of goal-directed actions. ${ }^{7}$ The neuroscientific discovery of processes of mirroring on which this claim rests is that the same brain areas are active during the execution and the observation of goal-directed actions. This claim seems appealing because it parsimoniously restricts the representational resources necessary for the perception of others' goal-directed actions to the resources necessary for the execution of goal-directed actions (Knoblich [2008]; Prinz [1990]).

The claim that resonance is a necessary condition for the perception of another's goaldirected action seems to rest on the acceptance of an overly strong version of the motor theory of perception, according to which the perception of another's goal-directed action just consists in motor simulation, that is in the simulation of the agent's bodily movements, without executing them. On this assumption, one is able to perceive others' goal-directed actions only to the extent that one is able to simulate them with one's own motor system. This assumption seems to fly in the face of the evidence that we can perceive many goal-directed actions which we cannot motorily simulate, such as a snake's crawl or a bird's flight, or that very young infants, whose motor system is not yet mature, also show sensitivity to goal-directed actions they are not able to perform.

This is demonstrated empirically in studies on infants' comprehension of goal-directed actions. Skerry, Carey and Spelke ([2013]) explore how 3-month-old infants (who can't

\footnotetext{
${ }^{7}$ Another alternative interpretation of the role of resonance is due to Gallagher (Gallagher [2007]), according to whom 'simulation' is a person-level concept applicable to conscious phenomena, and not to the sub-personal, resonance, level. Therefore, mirroring cannot constitute 'implicit' simulation. He suggests instead that mirroring is part of an enactive process that contributes to the perceptual access to the intentions of others. Gallagher's position, in my view, belongs to the mechanisms issue, that is the question of what mirroring is a mechanism for, and the question of what the underlying mechanisms of social perception are. I disagree with Gallagher on the characterization of the processes involved in social perception, and in particular on their enactive nature - though I must leave in-depth engagement with Gallagher's important work for further research.
} 
themselves reach and grasp objects) already encode actions in terms of goals and physical constraints. One prediction of the resonance hypothesis is that understanding of action goals should be limited to the ones that have been experienced in the first-person. Contrary to this prediction, Skerry et al. show that 3-month-olds can understand actions that are kinematically distinct from actions they have experienced.

In experiments 1 to 3 , infants were assigned to three categories: infants who manipulated objects with adhesive Velcro mittens, infants who manipulated objects with non-adhesive Velcro mittens, and infants who underwent no training. Afterwards, all infants were habituated to watching an agent wearing Velcro gloves reaching over a barrier to grasp an object. After habituation, the barrier was removed and infants saw either an inefficient, arched reach toward the object, or a straight direct reach. Infants who had training with Velcro mittens looked longer at the inefficient arching reach, while infants who had ineffective training with non-adhesive Velcro mittens and infants with no training looked longer at the new direct path to the object.

According to the experimenters, this shows that infants are able to generalize their experience to actions at a level of abstraction that goes beyond the kinematics of actions they have experienced themselves.

Resonance-based views are also incompatible with some available experimental evidence on non-typical development. If perceptual resonant processes underlie the understanding of actions, then they seem prima facie well suited to play a double role: they support both the understanding and the perception of actions. But these are distinct capacities. Indeed, resonance-based views tend to conflate two ways of apprehending actions: social 
perception involves early detection of the social features of the world, before further elaboration that includes conceptualization in terms of mental states, and attribution of mental states to others. For example, as shown in this section, social perception comprises mechanisms for the distinction between goal-directed and non-goal directed actions within the broader class of movements ${ }^{8}$.

Social understanding, on the other hand, includes processes involved in mindreading, the ability to understand others' behavior as being caused by mental states (such as intentions), to interpret others' actions via an appeal to the underlying mental states, and to attribute these states to them. I thus follow Catmur ([2015]), who, in a paper criticizing the appeal to mirroring as a route to intention understanding, highlights the importance of this distinction and writes that we need a working definition of the contrast between action perception and intention understanding in order to make empirical progress $(\mathrm{p}$. $432)^{9}$.

For example, subjects with autism spectrum disorder have been shown to make the same saccades and to exhibit the same visual tracking of animated displays of geometric figures performing goal-directed actions as neurotypical subjects, even if they are

\footnotetext{
${ }^{8}$ Even if it does not entail the identification of the specific goal towards which the movement is directed. In this respect the perception of goal-directed actions is similar to the perception of agency in being binary: some movements are either goal-directed or not goal-directed, just as some entities are either animated or not animated.

${ }^{9}$ The conflation of these two aspects of social cognition is not limited to resonant theories, though. For example, Rutherford and Kuhlmeier write that the study of social perception includes, inter alia, the study of biological motion, attribution of intentionality, and recognition and interpretation of goal-directed action (Rutherford and Kuhlmeier [2013], p. 3). It seems thus that the difference between social perception and social understanding is about the subject matter (intentional actions vs. beliefs and desires) and not mainly about the underlying processing (perceptual vs. cognitive).
} 
impaired in the attribution of intentions, thus showing a disconnection between perception and judgment (Rutherford, Pennington, and Rogers [2006]). It could be that their perceptual abilities involved in the perception of goal-directed actions are intact, even in the absence of a spontaneous judgment and attribution of intentionality to the displays. The ability to perceive others' goal-directed actions need not rest on the ability to execute or simulate them.

\section{WHAT ARE THE NEURAL CORRELATES OF SOCIAL PERCEPTION (WITHOUT RESONANCE)?}

Further evidence for the necessity of distinguishing social perception and social understanding comes from the available body of neuropsychological evidence. This evidence supports the proposal that goal-directed actions are perceived either before resonance takes place or in the absence of resonance.

Such a claim rests on the exploration of the neural basis of the perception of goal-directed actions: the areas that plausibly contribute to the perception of goal-directed actions are non-resonant. This special role of the STS for perception is noted in the philosophical literature (Spaulding [2013]), but it has not been thoroughly discussed by philosophers. Empirical research emphasizes the central role of the STS for the social perception of goal-directed action (Hein and Knight [2008]; Jellema and Perrett [2007]; Molenberghs, Brander, Mattingley, and Cunnington [2010]; Zilbovicius et al. [2006]; Frith and Frith [2003]; Gallagher and Firth [2003]; Watson et al. [2014], Deen et al. [2015]). The right posterior area of the STS is sensitive to the relation between an observed motion and the environmental constraints on the motion (Saxe, Xiao, Kovacs, Perrett, and Kanwisher 
[2004]). The STS in both humans and monkeys activates for biological movements (both goal-directed actions, such as grasping, and actions directed to other conspecifics, such as lip- smacking), but also for static images of faces and bodies (Allison et al. [2000]). It also activates for animated motion even if the agent is a geometrical entity (Gao, Scholl, and McCarthy [2012]). Some cells in the lower bank of the STS respond to bodily movements directed at objects or locations (Perrett et al. [1989]). It thus seems that the STS plays a central role in perception of goal-directed movements, alongside with the perception of other social properties.

Importantly, the STS does not contain mirror neurons, that is neurons active in both the execution and the perception of actions. It only contains cells that respond to the perception of actions executed by agents (both conspecific and non-biological agents). In non-human primates, the STS sends reciprocal projections to the inferior parietal cortex $(\mathrm{PF})$, which is in turn reciprocally connected to the premotor cortex area F5, where mirror neurons were first discovered. However, there are no direct connections between areas F5 and the STS (Jellema and Perrett, 2007; Keysers and Perrett [2004]). So, whatever role mirroring plays in social perception, in and of itself, the STS cannot underlie resonance. But it nonetheless plays a prominent role in the perception of goal-directed actions, and more broadly social perception. This evidence provides additional support for the claim that resonance is not necessary for perceiving goal-directed actions.

A paper by Deen et al. ([2015]) explores the functional organization of the STS in more detail. While the STS activates both for people reading stories that involve mental states (task of mentalizing), and for people seeing goal-directed actions, the study found that there is no overlap between responses to theory of mind tasks and observation of 
biological motion: response to biological motion was anterior to the response to theory of mind tasks (which activated the temporo-parietal junction). This further speaks against the conflation of social perception and understanding.

Still, as the authors of the study themselves remark, one of the main difficulties is to map representations into the processes underlying them (perceptual or cognitive). They write that 'another general limitation of this study is that while we would like to identify regions of the STS that are involved in specific cognitive and perceptual processes, we instead use the proxy of identifying regions of the STS that respond in a given task contrast, as in any fMRI study' (Deen et al. [2015]).

Three issues might undermine the appeal to the STS as playing a central role of the perception of goal-directed actions: these issues stem from the fact that the STS is a highlevel perceptual area and therefore ill-suited to distinguish between what counts as perceptual and what counts as post-perceptual. These issues, however, can be solved.

A first worry concerning the STS is that this brain area seems to become active in so many situations that it has been called 'the chameleon of the human brain' (Hein and Knight [2008]).

Apart from the role in some mentalizing tasks, the various activations of the STS can be grouped in two broad families: one concerned with social perception (including perception of goal-directed actions, biological motion, faces and expressions) and another concerned with the integration of different sensory modalities. STS is thus not a primary visual area, but one that underlies (comparatively) higher-level visual processing. 
Cross-modal integration, however, is not yet post-perceptual processing, or conceptual thought. Thus, the fact that an area is involved in cross-modal integration does not show that the area is dedicated to post-perceptual processing, unless one simply assumes without any argument that cross-modal integration cannot occur within perceptual processing. This assumption, however, would be inconsistent with the increasingly widespread evidence that perception is intrinsically multi- and cross-modal (Calvert, Spence and Stein [2004]). In fact, binding several features of an object is typical of perceptual processing. It is uncontroversial that the visual system can bind several attributes (for example its size, shape, color, orientation and motion) of an object (for example a dog) into a single visual percept. It further seems uncontroversial that binding is also what underlies the creation of a cross-modal percept by integrating the visual representation of a dog and either the auditory experience of the barking of the dog or the tactile experience of touching its fur. So, while future research on the role of the STS is undoubtedly necessary for better understanding how multi-modal integration underlies social perception, there is little reason to worry so far about the perceptual status of representation in STS.

Further supporting the claim that the representation of action in STS is perceptual, there is a clear difference between perceiving a goal-directed action performed by a human agent (for example locomotion), even if doing so indeed requires binding, and thinking about it or even imagining it. The STS is active when one perceives a goal-directed action executed by another, but it is not active when one imagines another's goal-directed action. It is active, however, when someone reads stories involving mentalizing. But, as we already saw, there is no functional overlap between these activations (Deen et al. 
[2015]). While I am not using this point to conclusively demonstrate that we perceive goal-directed actions, it does lend some support to my claim that activity of the STS is associated with high-level social perception.

A second worry is that what findings of experiments on the STS really show is that the STS is selectively activated for biological movements. If so, this would be consistent with the view that what is being encoded by the output of the activity of the STS is not the representation of goal-directed actions per se, but instead the kinematic features of an agent's movements in relation to her environment, provided that the agent is a biological agent. In other words, the STS would be selectively activated by the processing of movements which obey the same biological constraints as the movements that humans and animals can perform. But it would only encode the low-level kinematic features of these biological movements, not the high-level property of actions of being goal-directed.

To answer this worry, I appeal to the evidence that the STS does not only respond when subjects see goal-directed actions performed by biological agents, but also when they see goal-directed actions performed by non-biological agents, such as geometrical shapes, which execute motions which do not satisfy the constraints of biological movements (Gao, Scholl, and McCarthy [2012]). Gao, Scholl and McCarthy's study explores the neural correlates of the perception of animacy and goal-directed actions (which in the study is described, somewhat misleadingly, as 'intentionality') in the right posterior STS, or pSTS. The experimental design consists in manipulating perceptual cues for goaldirected actions, while maintaining constant perceptual cues for animacy. Practically, this is done with a 'wolf chasing sheep' display, in which a colored disk (the wolf) chases one of the other two colored disks (the sheep and the 'bystander'). Different conditions were 
tested: a) non-goal directed displays with patterns of motions where only low-level nonsocial motion properties were present; b) displays where the 'wolf' would not change goals but chase one target consistently; c) displays where the 'wolf' would change its target. The Changing Intentions condition gave rise to stronger activation in the right pSTS (posterior STS) and adjacent right LOTC (lateral occipitotemporal cortex) than did the other conditions (Gao, Scholl, and McCarthy [2012]).

Statistically speaking, we more frequently see goal-directed actions executed by biological agents, whose movements are in accordance with the constraints of biological motions, than goal-directed actions performed by non-biological agents. Nevertheless, activity of the STS can be triggered by the detection of goal-directed actions performed by non-biological agents. This tells against the claim that STS only responds to low-level kinematic features, and supports the view that it enables us to perceive goal-directedness.

A third issue is that someone could claim that the perception of low-level sensory features was sufficient for prompting activations of the STS. This claim, however, is in contrast with the known patterns of activations of this area: the STS activates for biological motions, goal-directed actions, emotional expressions, voices, but not for random non goal-directed motions, colors, orientations, ... For instance, in the Changing Intentions condition, right pSTS showed strong selective activation in the goal-directed condition, but not in the condition where subjects observed only random non-goal directed motion (Gao, Scholl, and McCarthy [2012]). ${ }^{10}$ The effect just 'pops out' in

10 The authors write ' $[\mathrm{o}] \mathrm{n}$ the basis of these results $[\ldots]$ we conclude that the right pSTS is engaged in social perception, as distinct from other non-social factors. This same lesson has been drawn from many other studies, but it has been challenging to support such claims given that the relevant social vs. nonsocial conditions have always differed in so many other correlated ways. Here we submit that this contrast in the 
vision.

At this point, one might be tempted to object that this is all well and good, but since geometrical figures do not really have goals, this undermines my thesis: We cannot possibly see goals. I offer the following two-tiered rejoinder to this conceptual objection.

First, we need to be clear what 'goal' means. As I indicated in section 1, 'goal' in this context should not be construed as an internal mental representation, but instead as the external outcome of a movement. Once again, my thesis is not aimed at establishing that we perceive goal-directed actions, when goal means a private mental state. I use goal in the sense of a publicly available outcome of a movement, where the outcome is an event. One reason this distinction is important, as I also pointed out earlier in the discussion of the putative role of resonance, is that we should be careful to distinguish between different scopes of social perception. The stronger version proposes that we perceive mental states as such (intentions qua mental states, or goals as internal and not external goals). According to the more moderate version that I wish to defend, what can be represented in perception is not limited to the processing of low-level sensory features (such as the kinematic properties discussed above).

Second, it is necessary to clarify what 'sees' means. Many philosophers employ the term in a factive sense: One can only see what actually exists or is the case. If 'sees' is read as factive, then - for instance - the claim that we see goal-directed actions in Heider and Simmel displays would be false. I have no qualms with those who wish to use 'sees' as a

present study is especially compelling, as our use of the chasing displays allowed us to rule out these competing factors, varying social information while holding nearly every lower-level property constant.' (Gao, Scholl, and McCarthy [2012]). 
success term. But there is a perfectly good, interesting sense in which we do see goaldirected actions in the relevant sorts of displays. Perceptual displays serve as proxies for the spontaneous formation of (sometimes illusory) perceptual judgements, and allow for fast shortcuts for navigating the social world. For example, imagine being transported to Flatland, where squares and spheres do have mental states. Perception, as a psychological process, treats as goal-directed actions all movements toward objects when certain cues are present. When we reflect, we may think otherwise. This relates to the also commonly accepted view that, actually, not all perception is factive: seeing an $\mathrm{x}$ as an $\mathrm{F}$ does not imply that the $\mathrm{x}$ is an $\mathrm{F}$ (Dretske [2000]).

\section{FROM NEURAL CORRELATES TO THE EXPERIENCE OF GOAL-DIRECTED ACTIONS}

At this point, a philosopher interested in the contents of perceptual experience could object that the neuropsychological evidence presented in the previous section, while interesting, does not really bear very directly on the question of what a person can see (as opposed to cognize), because none of the evidence is at the personal level. Does the data about processing really tell us anything about the subject's phenomenal experience?

While many studies discussed above do not directly tap into the subjects' phenomenal experiences, there is empirical evidence that the perception of goal-directed action gives

rise to a strong phenomenal experience as opposed to actions that are not goal-directed. For instance, in Gao et al.'s studies, activation in the STS was highest in the goal-directed condition, but not in other conditions. 
The presence of a goal-directed action (that is the presence of 'chasers') influenced the subjects' performance, even when the goal-directed stimulus was irrelevant to the task (Gao, McCarthy and Scholl [2010]). In another paper, the same authors explore the presence of another cue of goal-directedness action, which they call the 'wolfpack effect': when a randomly moving set of geometrical figures persistently keep on pointing to the same moving disc as if they were chasing it (Gao, McCarthy and Scholl [2010]).

But independently of such empirical evidence, why suppose that we perceive goaldirected actions in the first place? I think it is worth making a fairly intuitive experiencebased case for such a claim. The striking fact that we may experience the illusion of intentionality in the presence of a goal-directed action performed by a non-biological agent without intentions is strong evidence that our visual experience of goal-directed action is indeed perceptual. Take the case of the famous animated displays of Heider and Simmel: geometrical stimuli are seen as chasing each other and the viewers have an irresistible drive to describe the displays in mentalistic terms (Heider and Simmel [1944]). We reflectively know that geometrical figures cannot truly have goals. They do not possess the kind of structure folk think can bear mental states - they do not have a brain. Yet we cannot stop ourselves from automatically ascribing mental states to them and having the impression of mentalistic goal-driven entities despite our best efforts. Here is a speculation about what happens in these cases: we see goal-directed actions and, on the basis of our perceptual experience, we immediately and non-reflectively formulate the perceptual judgement that the entity has an intention or mentalistic goal. This judgment is based on a perceptual experience, and so can - and typically will - soon conflict with our reflective judgment that geometrical stimuli can't have goals. The 
hypothesis that these are instances of perception is an initially plausible one. If it is further supported by independent empirical evidence, then we should adopt it - absent powerful arguments for competing views.

Such considerations do, in my opinion, help make the case that we (and not just some inaccessible regions of our brain) perceptually represent goal-directed actions. Yet I grant that the question of whether the evidence about the neural substrates correlate with a perceptual experience of goal-directed actions remains difficult to answer. It hinges on the notorious issue of the connection between the neural and experiential levels. While my aim is not to provide an ultimate reply to this issue, I want to speculatively suggest, to conclude this section, that there is an indirect way to explore a positive answer, which will need more testing and confirmation, but is an initial step in the right direction.

The indirect way goes through the investigation of perceptual adaptation to goal-direct actions involving lifting of, and placing, of heavy and light objects (Barraclough, Keith, Xiao, Oram, and Perrett [2009]). Adaptation studies are used to manipulate subjects' perceptual experiences. For example, after adaptation to a grating with lines tilted toward the left, the subject experiences a subsequent grating with vertical lines as if the lines were tilted toward the right. Adaptation therefore provides some guidance to the contents of perceptual experiences (Gibson and Radner [1937]; for discussion see Block [2014]).

In the experiment on placing and lifting actions, a human hand grasped a block and lifted it. The test involved two kinds of actions: grasping and placing. Subjects passively observed the action and their impressions of lightness or heaviness were manipulated by the ratio of the reach and withdraw phases. When the reach phase was longer than the 
withdraw phase, subjects consistently perceived the object as lighter. When the withdraw phase was longer than the reach phase, subjects consistently perceived the object as heavier. The subjects first adapted to either a light or a heavy object action, and then they watched an action involving an intermediate weight action. They responded by using a rating scale from $100 \mathrm{gr}$ to $900 \mathrm{gr}$ to indicate the perceived weight of the object. Adaptation propagated across kinds of actions (adapting to the perception of grasping objects had an influence on the perception of placing objects). Subjects, who visually adapted to actions involving a heavy object, saw the subsequent actions as involving lighter objects, and vice versa (Barraclough et al. [2009]).

Two conditions were tested: for the same kind of actions (placing-to-placing) and for different kinds of actions (placing-to-grasping). In placing-to-placing, after adapting to a hand placing a heavy object, subjects experienced the subsequent placing display as involving a lighter object. In placing-to-grasping, after adapting to a placing action, subjects saw a subsequent grasping action as involving heavier objects than the object they had adapted to.

One worry is that experiments on perceptual adaptation alone cannot establish that goaldirected actions are perceived, because the subjects' reports are based on judgments of the relative weights of the objects involved. But the judgment might not faithfully mirror the perceptual content of the experience. In order to answer to this worry, the authors also performed single-cell recordings in the monkey's STS during observation of goaldirected movements and found that cell responses were sensitive to the presentation of the action and the presence of the object. Thus, the object is an integral part of the goaldirected action whose perception cannot be reduced to the perception of a spatiotemporal 
trajectory alone. Still, one cannot conclude that the same arises for the human subjects as well.

Someone else could use the same evidence to claim the opposite and say that the content of perceptual experiences is actually finer-grained than just being a goal-directed action. The reason is that the experiment is not about the perception of goal-directed actions per se, but it is instead about the visual experience of the heaviness and lightness of objects as part of the perception of a goal-directed action. Therefore, the perceptual content in this case is more fine-grained than the representation of being a goal-directed action.

It is plausible, however, that in order to perceive an action as involving lighter or heavier objects one has to experience goal-directed actions, and not simple movements. Indeed, it would seem incoherent to assume that one represents the property of an action to involve lighter or heavier objects, while merely representing the kinematic properties of the agent's movements and not its goal-directedness. Moreover, this option is still a case of the rich perceptual content view, and opens up another possibility for the relation between resonance and perception of goal-directedness that I will explore in the following section.

\section{THE DEVELOPMENT OF THE PERCEPTION OF GOAL-DIRECTED ACTIONS: A POSSIBLE NEW ROLE FOR RESONANCE}

In this section, I further explore the relation between the perception of goal-directed actions, resonance, and phenomenal experience. Rather than criticize extant resonance- 
based views, however, I make a suggestion about the connection between the two phenomena. This suggestion fits with the hypothesis that mirroring underlies associated learning for the understanding of actions performed by others (this hypothesis has been suggested by Calvo-Merino et al. [2005] and Press [2011]). I extend this proposal to hypothesize that mirroring might also play a role in fine-tuning our perceptual experiences of others' movements involved in goal-directed actions. To buttress this proposal, I will rely on two intriguing empirical results: one in infant perception research and another in the investigation of perceptual experiences of aplasic patients (patients born without arms).

The first experiment is an exploration of the perception of goal-directed behavior in infants (6- to 8- months old) (Southgate, Johnson, and Csibra [2008]). Infants observed a display where three walls (functioning as obstacles) and a ball in front of one of the walls were present. They were first familiarized with a normal efficient goal-directed action, whereby a hand removed an obstacle before reaching for a ball. After familiarization, two conditions were tested. In the first condition, infants observed the hand remove the obstacles before reaching for the ball via a biologically plausible motion. In the second condition, instead of removing the obstacle, the arm to which the hand was attached undulated (in a snake-like fashion) in a biologically implausible manner to reach the ball despite the obstacles. Infants looked longer in the first condition than in the second. The results show that infants were more surprised ${ }^{11}$ (where surprise was measured by longer looking times) to see the hand perform a biologically plausible but less efficient goaldirected action than a more efficient but biologically implausible goal-directed action

11 Longer looking time is considered to express attention and hence surprise in pre-verbal infants. Surprise indicates a discrepancy between the expected result and the witnessed result (Aslin [2007]). 
(inconsistent with biomechanical principles). In these displays, motor similarity between the infants' motor repertoire and the observed action did not constrain infants' perception. Infants were not surprised to see biologically impossible motions, such as a hand 'undulating' like a snake when avoiding obstacles to grab an object. The authors interpret this result as evidence against the view that action understanding is rooted in resonance and motor experience.

Why are infants in Southgate et al.'s experiments not surprised by violations of biological motion, while adults are? There is evidence that infants use the presence of a hand to detect the presence of a human agent (Woodward et al. [2003]), so the question is whether infants know 'how hands work'. If they represent the hand as the presence of a human agent, it might be that at this early age (at 6 to 8 months old), infants' motor repertoire is not sufficiently developed to allow for the simulation of this kind of hand motion. Still, they perceive goal-directed actions.

While the mechanisms underlying the perception of goal-directed actions might be present early, motor expertise might develop later as infants come to be exposed to a greater variety of goal-directed actions specifically performed by human agents. It seems plausible that infants are endowed with basic mechanisms for perceiving goal-directed actions, even if they do not yet have a precise biological motion scheme.

Much before human infants can successfully execute goal-directed actions such as locomotion, reaching for and grasping a target, early perceptual mechanisms make them attuned early on to the perception of a variety of goal-directed actions performed by a variety of non-human agents. For example, they can see a bird fly and a snake crawl (as 
the hand in Southgate et al.'s displays). Only when they become able to execute biological movements which are accessible to them can they learn about the biomechanical constraints which sort out biological movements accessible to humans from biological movements only accessible to birds or snakes, and also from nonbiological movements neither accessible to humans nor to snakes and birds.

If so, then resonance is likely to play a role in fine-tuning the perception of movements involved in the execution of goal-directed actions, rather than a foundational role in enabling the perception of goal-directed actions (see also Skerry, Carey and Spelke ([2013]) for additional evidence on infants' initial detection of goal-directed actions and the enriching role of first person motor experience).

The ability to map another's goal-directed action onto one's own motor repertoire is not necessary for the perception of another's action as being goal-directed. But it may nevertheless play a role in shaping the fine-grained perceptual experience of others' movements.

A potential objection to this proposal is that Southgate et al.'s experiment might not tap directly into the experienced contents of perception. There is, however, a paradigm that explores the way in which (bodily) movements are experienced in perception: the apparent body motion paradigm, which shares similarities to the experiment above. In research on apparent body motion (Shiffrar and Freyd [1993]; Shiffrar [2008]), people perceive the longer, but anatomically plausible path, rather than the shortest, but anatomically implausible path, when seeing a hand and arm action.

In a typical experiment, subjects view flickering pictures or videos of an actor performing 
a movement: the movement can be perceived either as a short movement that does not respect biological constraints (for example a hand passing through a head) or as a longer movement that respects biological constraints (for example a hand rotating around the head). In these displays, biological plausibility constrains the perception of movements. Subjects consistently consciously perceived a hand wrapping around a head, rather than a hand passing through a head, or an arm wrapping around another arm instead of passing through.

Shiffrar's experiments were tested on aplasic subjects (subjects born without arms): one aplasic subject experienced the phantom limb phenomenon, the other did not. The first subject experienced the longer and biologically plausible path in perception, while the second subject experienced the shorter and biologically implausible path (Funk, Shiffrar, and Brugger [2005]). Both subjects know how arms usually move, even if they never had arms them themselves. Still, their phenomenal experiences were different, because in one case, there was resonance between another's real arms and the subject's phantom arms, and in the other case, there was not. So resonance and motor expertise might contribute to phenomenal experience through the fine-tuning of the perception of an agent's biological movements during the observation of her goal-directed actions.

To sum up, some experimental evidence points toward the idea that resonance does indeed play a role in the perception of goal-directed actions. It makes the content of the initial set of perceptual representations finer-grained, as the evidence of a contrast between the perceptual representations with and without resonance in aplasic subjects shows. This suggests a new role for resonance -- one that has been mostly overlooked in the literature on the perception of others' actions by authors who claim that resonance is 
necessary for perceiving others' goal-directed actions. While resonance is not necessary for perceiving goal-directed actions, it may nonetheless contribute significantly to the perception of others' movements in the execution of goal-directed actions. Investigating this proposal is a promising avenue for further research.

\section{CONCLUSION: SEEING GOAL-DIRECTEDNESS OF ACTIONS AND SOCIAL PERCEPTION}

Let us take stock. I have considered cognitive neuroscientific evidence for the view that the goal-directedness of others' actions can be represented in perception. I have considered and rejected the appeal to motor resonance as a necessary condition for the perception of goal-directed actions as such. I have further argued that the neurophysiological evidence strongly suggests that the STS plays a central role in the perception of goal-directed actions. These two views are closely related, since the STS does not contain mirror neurons, that is cells that underlie resonance because they fire both during the execution and the perception of goal-directed actions. The available empirical evidence also shows that the STS sends information to areas containing mirror neurons, but does not receive information from them. I proposed a hypothesis which accounts for all this data: Resonance may contribute to the further elaboration of the information processed by the STS. Further evidence from studies on perceptual adaptation and on the experiential and behavioral differentiation between non-goaldirected and goal-directed motions shows that activations of the STS is correlated with the phenomenal experience associated with the perception of goal-directed actions.

The picture that emerges is one in which a distinction is drawn between social perception 
and social understanding. Distinguishing mechanisms and processes underlying perception from those underlying understanding, however, does not necessarily create an insurmountable gap between the two. Understanding another's goal-directed action may require mapping her action onto one's own ability to act. Further research is needed to explore the rich connections between social perception and social understanding at the fully conceptual level. The hypothesis concerning the perception of goal-directed actions presented in this paper is but a first step in describing and outlining a distinctive and overlooked aspect of human visual perception and social cognition, social perception, the ability to visually detect and track properties related to other animated beings (human and non-human).

Future research on social perception will shed more light on this distinctive feature of social cognition. There are at least four possible avenues worth exploring. The first issue concerns the reach of social perception: which social properties can be represented in perception? While in this paper I have made a case for the perception of goal-directed actions, there are other potential candidates: for example, animacy and being a living being (as opposed to being an inanimate object or an artifact), emotional expressions (and in particular expressions for the six basic emotions), age, gender, social relations (being dominant or subordinate). In order to explore this issue, we might need to appeal to a variety of empirical means: from studies on the neural correlates of high-level perception to perceptual adaptation studies, from studies on the timecourse of social perception to studies on non-typical development and impairments. The exploration of the reach of social perception is connected with the question of the ontology of mental states, and in particular with the issue of whether mental states are embodied in their behavioral 
manifestations. ${ }^{12}$ While I set aside the ontology of mental states in this paper, I agree with direct social perception approaches that it is ultimately central for understanding the power of social perception (Krueger [2012]). If the embodiment hypothesis is correct then social perception might indeed extend to mental states and others' emotional states or intentional actions beyond their behaviors. Given the highly controversial status of the embodiment hypothesis, I prefer to remain neutral on this issue here.

The second issue concerns the function of social perception in social cognition. One particularly interesting question is the connection between social perception and action: is there a direct route from social perception to action that bypasses understanding? For example, by seeing someone's emotional expression we could formulate an immediate, automatic reaction, even if we haven't yet conceptualized the emotional expression (such as a defense reaction when seeing fear or anger). There is some evidence on automatic motor preparation in the case of emotional expressions of fear (Borgomaneri, Gazzola, and Avenanti [2012]). Similar studies could be done for goal-directed actions.

The third concerns the relation between social perception and social understanding (which includes mindreading). Whether social perception can support some forms of social understanding, as direct perception and resonance theory claim, is still up for grabs. One hypothesis is that the function of social perception is just to provide contents for further processing in higher cognition for mindreading and understanding, and to connect experience with fast and automatic reactions. Another hypothesis is that social perception also has a role in the understanding of social properties and of mental states. Further evidence and arguments are needed to decide between these two options.

\footnotetext{
${ }^{12}$ I am thankful to an anonymous reviewer for pointing out the question of embodiment.
} 
A final area for future research concerns psychopathological disorders. ${ }^{13}$ Some impairments within the social domain might be due to impairments in mindreading, others might be due to impairments in social perception (for example the inability to see actions as goal directed) - and some might be due to impairments of both sorts. Thus, an alternative to the interpretation of autism spectrum disorders briefly sketched above (p. 14) is that some troubles in understanding others' intentions might be due to impairments to perceptual level processing of actions, as opposed to high-level cognitive understanding in theory of mind (see Gallese and Rochat [2018]). Further work on impairments of social perception should shed lights on these issues.

\section{ACKNOWLEDGEMENTS}

I am grateful to Pierre Jacob, Frédérique de Vignemont, Michael Murez, Nico Orlandi, and Nick Shea for comments and help; to the audiences in Tübingen, Paris, and Bochum for their useful feedback; and to the anonymous reviewers who helped me improving this paper.

Faculty of Philosophy University of Oxford

Oxford, $U K$ joulia.smortchkova@philosophy.ox.ac.uk

\section{REFERENCES}

Allison, T., Puce, A., and McCarthy, G. [2000]: 'Social perception from visual cues: role of the STS region', Trends in Cognitive Sciences, 4(7), pp. 267-78.

\footnotetext{
${ }^{13}$ I am grateful to an anonymous reviewer for suggesting this potential area of research for social perception and for the autism spectrum disorder example and reference.
} 
Barraclough, N. E., Keith, R. H., Xiao, D., Oram, M. W., and Perrett, D. I. [2009]:

'Visual adaptation to goal-directed hand actions', J. Cognitive Neuroscience, 21(9), pp. $1806-20$.

Block, N. [2014]: 'Seeing-As in the Light of Vision Science', Philosophy and Phenomenological Research 89 (1), pp. 560-72.

Borgomaneri, S., Gazzola, V., Avenanti, A. [2012]: 'Motor mapping of implied actions during perception of emotional body language', Brain stimulation, 5(2), pp. 70-6.

Burge, T. [2010]: Origins of Objectivity. Oxford: OUP.

Butterfill, S. [2015]: 'Perceiving expressions of emotion: What evidence could bear on questions about perceptual experience of mental states?', Consciousness and Cognition, 36, pp. 438-51.

Calvert, G., Spence, C., and Stein, B. E. [2004]: The handbook of multisensory processes, Cambridge, MA: MIT press.

Calvo-Merino, B., Glaser, D. E., Grèzes, J., Passingham, R. E., and Haggard, P. [2005]:

'Action observation and acquired motor skills: an FMRI study with expert dancers', Cerebral Cortex, 15(8), pp. 1243-49.

Carey, S. [2009]: The Origin of Concepts, Oxford: OUP.

Carruthers, P. [2015]: 'Perceiving mental states', Consciousness and Cognition, 36, pp. 498-507. 
Catmur, C. [2015]: 'Understanding intentions from actions: Direct perception, inference, and the roles of mirror and mentalizing systems', Consciousness and Cognition, 36, pp. $426-33$.

Davidson, D. [1963]: ‘Actions, reasons, and causes', Journal of Philosophy 60 (23), pp. $685-700$.

Deen, B., Koldewyn, K., Kanwisher, N., and Saxe, R. [2015]: 'Functional organization of social perception and cognition in the superior temporal sulcus', Cerebral Cortex, bhv111.

Dretske, F. [2000]: 'Conscious Experience', in Perception, Knowledge and Belief: Selected Essays, Cambridge: CUP.

Frith, U., and Frith, C. D. [2003]: 'Development and neurophysiology of mentalizing.'Philosophical Transactions of the Royal Society of London B: Biological Sciences, 358(1431), pp. 459-73.

Funk, M., Shiffrar, M., and Brugger, P. [2005]: 'Hand movement observation by individuals born without hands: phantom limb experience constrains visual limb perception', Experimental Brain Research, 164(3), pp. 341-46.

Gallese, V. [2009]: ‘Motor abstraction: A neuroscientific account of how action goals and intentions are mapped and understood', Psychological Research PRPF, 73(4), pp. $486-98$.

Gallese, V., and Rochat, M. J. [2018]: 'Forms of Vitality: Their Neural Bases, Their Role in Social Cognition, and the Case of Autism Spectrum Disorder', Psychoanalytic Inquiry, 38(2), pp. 154-64.

Gallagher, S. [2008]: 'Direct perception in the intersubjective context', Consciousness 
and Cognition 17 (2), pp. 535-43.

Gallagher, S. [2007]: 'Simulation trouble’, Social Neuroscience, 2(3-4), pp. 353-65.

Gallagher, H. L., and Frith, C. D. [2003]: 'Functional imaging of 'theory of mind", Trends in cognitive sciences, 7(2), pp. 77-83.

Gao, T., McCarthy, G., and Scholl, B. J. [2010]: 'The Wolfpack Effect Perception of Animacy Irresistibly Influences Interactive Behavior.' Psychological science, 21(12), pp. $1845-53$.

Gao, T., Scholl, B. J., and McCarthy, G. [2012]: 'Dissociating the Detection of Intentionality from Animacy in the Right Posterior Superior Temporal Sulcus', Journal of Neuroscience, 32(41), pp. 14276-80.

Gibson, J. J. and Radner, M. [1937]: 'Adaptation, after-effect and contrast in the perception of tilted lines. I. Quantitative studies', Journal of Experimental Psychology 20 (5), p. 453.

Goldman, A. I. [2013]: Joint Ventures: Mindreading, Mirroring, and Embodied Cognition, New York: OUP.

Grezes, J., and Decety, J. [2001]: 'Functional anatomy of execution, mental simulation, observation, and verb generation of actions: a meta-analysis', Human Brain Mapping, 12(1), pp. 1-19.

Heider, F., and Simmel, M. [1944]: 'An experimental study of apparent behavior', The American Journal of Psychology, pp. 243-59. 
Hein, G., and Knight, R. T. [2008]: 'Superior temporal sulcus_it's my area: or is it?', Journal of Cognitive Neuroscience, 20(12), pp. 2125-36.

Hawley, K. and Macpherson, F. (eds.) [2011]: The Admissible Contents of Experience, Hoboken: Wiley-Blackwell.

Hutto, D., Herschbach, M. and Southgate, V. [2011]: 'Editorial: Social Cognition: Mindreading and Alternatives', Review of Philosophy and Psychology 2 (3), pp. 375-95.

Jacob, P. [2009]: 'A Philosopher's Reflections on the Discovery of Mirror Neurons', Topics in Cognitive Science 1 (3), pp. 570-95.

Jacob, P. [2011]: 'The Direct-Perception Model of Empathy: a Critique', Review of Philosophy and Psychology, 2 (3), pp. 519-40.

Jeannerod, M. [1995]: 'Mental imagery in the motor context', Neuropsychologia, 33(11), pp. 1419-32.

Jellema, T., and Perrett, D. I. [2007]: 'Neural pathways of social cognition', Oxford Handbook of Evolutionary Psychology, p. 163.

Keysers, C., and Perrett, D. I. (2004). Demystifying social cognition: a Hebbian perspective. Trends in Cognitive Sciences, 8(11), pp. 501-7.

Kiverstein, J. [2015]: 'Empathy and the responsiveness to social affordances', Consciousness and Cognition, 36, pp. 532-42.

Knoblich, G. [2008]: 'Bodily and motor contributions to action perception', In R. L. Klatzky, B. MacWhinney, and M. Behrmann (Eds.), Embodiment, ego-space, and action 
(pp. 45-78), Hove: Psychology Press.

Krueger, J. [2012]: 'Seeing mind in action', Phenomenology and the Cognitive Sciences, 11(2), pp. 149-73.

Molenberghs, P., Brander, C., Mattingley, J. B., and Cunnington, R. [2010]: 'The role of the superior temporal sulcus and the mirror neuron system in imitation', Human Brain Mapping, 31(9), pp. 1316-26.

Noë, A. [2005]: Action in Perception. Cambridge, MA: MIT Press.

Pacherie, E. [2005]: 'Perceiving intentions', A Explicação Da Interpretação Humana,, pp. $401-14$.

Pacherie, E. [2011]: 'Nonconceptual representations for action and the limits of intentional control', Social Psychology, 42(1), pp. 67-73.

Perrett, D. I., Harries, M. H., Bevan, R., Thomas, S., Benson, P. J., Mistlin, A. J., Chitty, A.J., Hietan J.K., Ortega, J. E. [1989]: 'Frameworks of analysis for the neural representation of animate objects and actions', Journal of Experimental Biology, 146(1), pp. $87-113$.

Press, C. [2011]: 'Action observation and robotic agents: Learning and anthropomorphism', Neuroscience and Biobehavioral Reviews, 35(6), pp. 1410-18. doi:10.1016/j.neubiorev.2011.03.004

Prinz, W. [1990]: 'A Common Coding Approach to Perception and Action', In O. Neumann and W. Prinz (Eds.), Relationships Between Perception and Action - Current 
Approaches (pp. 167-201). Berlin, Heidelberg: Springer.

Proust, J. [2003]: 'Perceiving intentions', Agency and Self-Awareness, pp. 296-320.

Rutherford, M. D., Pennington, B. F., and Rogers, S. J. [2006]: 'The Perception of Animacy in Young Children with Autism', Journal of Autism and Developmental Disorders, 36(8), pp. 983-92.

Rizzolatti, G. and Sinigaglia, C. [2007]: Mirrors in the Brain: How our minds share actions and emotions. Oxford: OUP.

Rizzolatti, G., Fogassi, L., and Gallese, V. [2001]: 'Neurophysiological mechanisms underlying the understanding and imitation of action', Nature Reviews Neuroscience, 2(9), pp. 661-70.

Saxe, R., Xiao, D.-K., Kovacs, G., Perrett, D., Kanwisher, N. [2004]: ‘A region of right posterior superior temporal sulcus responds to observed intentional actions', Neuropsychologia, 42(11), pp. 1435-46.

Shiffrar, M. [2008]: 'The visual perception of dynamic body language', Embodied Communication in Humans and Machines, 95.

Shiffrar, M., and Freyd, J. J. [1993]: 'Timing and apparent motion path choice with human body photographs', Psychological Science, 4(6), 379-384.

Siegel, S. [2010]: The Contents of Visual Experience. Oxford: OUP.

Skerry A. E., Carey, S.E., and Spelke, E. S. [2013]: 'First-person action experience reveals sensitivity to action efficiency in prereaching infants', Proceedings of the 
National Academy of Sciences, 110(46), pp. 18728-33.

Southgate, V., Johnson, M. H., and Csibra, G. [2008]: 'Infants attribute goals even to biomechanically impossible actions', Cognition, 107(3), pp. 1059-69.

Spaulding, S. [2013]: 'Mirror Neurons and Social Cognition', Mind and Language, 28, pp. 233-57.

Spaulding, S. [2015]: 'On Direct Social Perception', Consciousness and Cognition, 36, pp. $472-82$.

Watson, R., Latinus, M., Charest, I., Crabbe, F., and Belin, P. [2014]: 'People-selectivity, audiovisual integration and heteromodality in the superior temporal sulcus', cortex, 50, pp. $125-36$.

Woodward, A., Sommerville, J. A., Guajardo, J. J., Malle, B., Moses, L. J., and Baldwin, D. [2003]: 'How infants make sense of intentional action', In Intentions and intentionality: Foundations of social cognition (pp. 149-171). Cambridge, MA: MIT Press.

Zilbovicius, M., Meresse, I., Chabane, N., Brunelle, F., Samson, Y., and Boddaert, N. [2006]: 'Autism, the superior temporal sulcus and social perception', Trends in Neurosciences, 29(7), pp. 359-66. 